\title{
LncRNA MEG3 Is Involved in Endothelial Differentiation of Human Bone Marrow-Derived Mesenchymal Stem Cells
}

\author{
Na Huang ${ }^{1, a}$, Rui Zhang ${ }^{1, b}$, Danyang Shi ${ }^{1, \mathrm{c}}$, Doudou $\mathrm{Li}^{1, \mathrm{~d}}$, Hongpeng $\mathrm{He}^{1, \mathrm{e}}$, \\ Hao Zhou ${ }^{1, f}$, Tong-Cun Zhang ${ }^{1, g}$, Nan Wang ${ }^{1, h}$
}

${ }^{1}$ Key Laboratory of Industrial Microbiology, Ministry of Education and Tianjin City, College of

Biotechnology, Tianjin University of Science and Technology

a541810464@qq.com, b910522085@qq.com, ${ }^{7} 754240170 @ q q . c o m$, d1242888739@qq.com, ehehongpeng@tust.edu.cn, ${ }^{\mathrm{f}}$ zhouhao@tust.edu.cn, ${ }^{9}$ tony@tust.edu.cn, ${ }^{\mathrm{h}}$ wn929@tust.edu.cn

Key words: long non-coding RNA, MEG3, Mesenchymal stem cells, Endothelial cells, Differentiation

Abstact. The role of long non-coding RNA in endothelial differentiation of MSCs remains poorly understood. In this study, the levels of several lncRNAs including MEG3, MALAT1, SNHG5, lnc00657 and TUG1, which are high expressed in endothelial cells, were detected in hMSCs and HUVECs by real-time PCR. The results showed that lncRNA MEG3 was significantly high expressed in HUVECs, compared with hMSCs. hMSCs treated with VEGF and bFGF can exhibit the increase of endothelial cell marker CD31 level and also the increase of MEG3, suggesting that MEG3 could be involved in the endothelial differentiation of hMSCs. Finally, blockdown of MEG3 using siRNA of MEG3 led to the decrease of CD31 expression and the inhibition of hMSCs differentiation into endothelial cells.

\section{Introduction}

More and more lncRNAs are involved in the transcriptional regulation of gene expression and exhibit important cellular function although having no protein-coding capability [1]. However, their roles in endothelial differentiation of MSCs remain poorly understood. Because of the multipotent differentiation potential and immunosuppressive properties, MSCs have been widely studied and used for regenerative medicine [2]. In our previous study, combination of VEGF and bFGF were used to induce hMSC differentiate into endothelial cells [3]. It has been reported that several lncRNAs including MEG3, MALAT1, SNHG5, lnc00657 and TUG1, are high expressed in endothelial cells [4]. In this study, we compared the expression of these lncRNAs between hMSCs and HUVECs and then preliminarily investigated the role of MEG3 in endothelial differentiation of hMSCs.

\section{Materials and Methods}

Cell Culture and Cell Differentiation Induction. hMSCs were kindly provided by Union Stem Cell and Gene Engineering Co. and HUVECs were kindly provided by Tianjin Medical University. Both hMSCs and HUVECs were cultured in Dulbecco's modified Eagle medium/Nutrient Mixture F12 (DMEM-F12, GIBCO) with 20\% FBS (Atlanta Biologicals), 1\% penicillin and streptomycin at $37^{\circ} \mathrm{C}$ with $5 \% \mathrm{CO}_{2}$.

For cell differentiation induction, hMSCs were cultured in medium (Endothelial Cell Growth Media Kits, Lonza) with $100 \mathrm{ng} / \mathrm{mL}$ VEGF (Sigma) and $50 \mathrm{ng} / \mathrm{mL}$ bFGF (Protech) for 7 days. These cells were observed under phase microscope.

Quantitative Real-Time RT-PCR (Qrt-PCR). qRT-PCR analysis were carried out as described previously [3]. The qRT-PCR was performed in an Applied Biosystems StepOne Real-Time PCR System (Applied Biosysterms). Data were shown as relative expression level after being normalized to18S. The PCR primer sequences are as Table 1. 
Table 1: Primers used for qRT-PCR

\begin{tabular}{lll}
\hline Gene & Primer sequence & \\
\hline 18S & F-CACGGGAAACCTCACCCGGC & R-CGGGTGGCTGAACGCCACTT \\
MEG3 & F-GAGTGTTTCCCTCCCCAAGG & R-GCGTGCCTTTGGTGATTCAG \\
CD31 & F-AGACGTGCAGTACACGGAAG & R-TTTCCACGGCATCAGGGAC \\
MALAT-1 & F-GTGATGCGAGTTGTTCTCCG & R-CTGGCTGCCTCAATGCCTA \\
TUG1 & F-TAACAGCCCTCCACTCCAGAT & R-AGGCACCAGCTTCAAAACCC \\
00657 & F-CAGGAGAATCGCTTGAACTT & R-CCCAAACACCCAATGAATAG \\
SNHG5 & F-GCCTCGACCCTGTATTGA & R-CAGGAGAATCGCTTGAACTT \\
\hline
\end{tabular}

RNA Interference. hMSCs were transfected with siRNA oligonucleotides using Turbo (Thermo) according to the manufacturer's instructions. The efficiency of RNA interference was determined by RT-PCR analysis.

Statistical Analysis. Data were presented as means \pm SD from at least three separate experiments. The significant difference was examined using the Student's $t$ test. The minimal level of significance was $P<0.05$.

\section{Results and Discussion}

The Levels of Lncrnas were Detected in Huvecs and Hmscs. The levels of lncRNAs including MEG3, MALAT1, SNHG5, 00657 and TUG1 were tested in HUVECs and hMSCs by RT-PCR. As shown in Fig. 1, the level of lncRNA MEG3 was higher in HUVECs, compared with hMSCs.

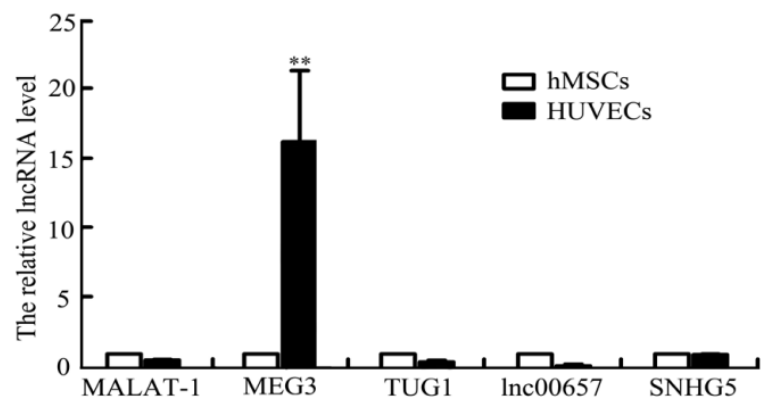

Figure 1. The levels of several lncRNAs including MEG3, MALAT1, SNHG5, 00657 and TUG1 were detected in HUVECs and hMSCs by real-time PCR.

VEGF and Bfgf Induced Hmsc Differentiation into Endothelial Cells. hMSCs were cultured in endothelial special medium containing $100 \mathrm{ng} / \mathrm{mL}$ VEGF and $50 \mathrm{ng} / \mathrm{mL}$ bFGF for $7 \mathrm{~d}$ and the morphological changes of these cells were observed under invert phase microscopy. As shown in Fig. 2(A), the control group cells exhibited spread and flat cell body, while the cells treated with VEGF and bFGF became spherical and round, an endothelial cell like phenotype. The mRNA levels of CD31 were upregulated in VEGF and bFGF-treated hMSCs and this increase was time-dependent (Fig. 2B).

A

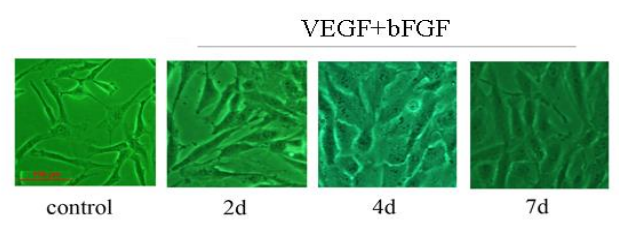

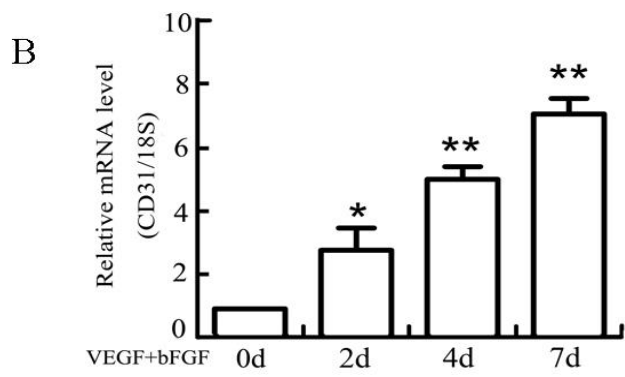

Figure 2. VEGF and bFGF induced hMSC differentiation into endothelial cells. (A) The morphological changes of hMSCs treated with VEGF and bFGF at $2 \mathrm{~d}, 4 \mathrm{~d}$ and $7 \mathrm{~d}$. (B) The mRNA levels of CD31 in VEGF and bFGF-treated hMSCs at 2d, 4d, 7d.

The Lncrna Levels of MEG3 Were Increased in VEGF and Bfgf-Treated Hmscs. The level of MEG3 was tested in VEGF and bFGF-treated hMSCs by real-time PCR. As shown in Fig. 3, 
VEGF and bFGF treatment enhanced the levels of MEG3 in time-dependent manner. These data showed that MEG3 could play a role in endothelial differentiation of hMSCs.

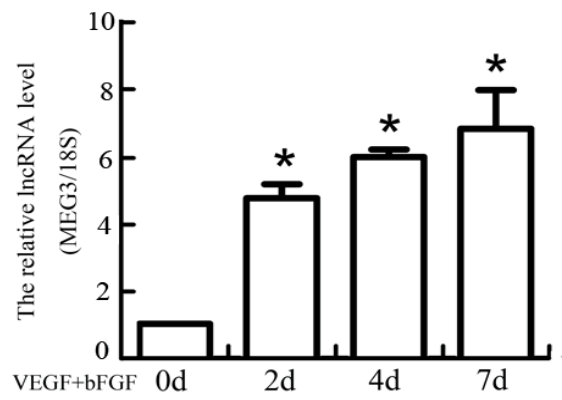

Figure 3. The levels of MEG3 were increased in VEGF and bFGF-treated hMSCs at $2 \mathrm{~d}, 4 \mathrm{~d}, 7 \mathrm{~d}$ by real-time PCR.

Downregulation of MEG3 Level Inhibited Human Mscs Differentiation into Endothelial Cells. Human MSCs were transfected with control siRNA or MEG3 siRNA. As shown in Fig. 4A, both siMEG3-2 and siMEG3-3 could significantly decrease the level of MEG3. hMSCs were transfected with control siRNA or siMEG3-2 in the presence of VEGF and bFGF for 7d and then the levels of CD31 were detected by real-time PCR (Fig. 4B). Knockdown of MEG3 decreased the mRNA level of CD31 stimulated by VEGF and bFGF, suggesting that MEG3 played an important role in endothelial differentiation of hMSCs.

A

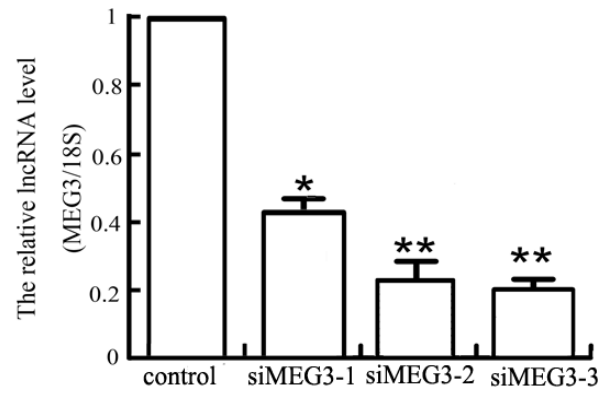

$\mathrm{B}$

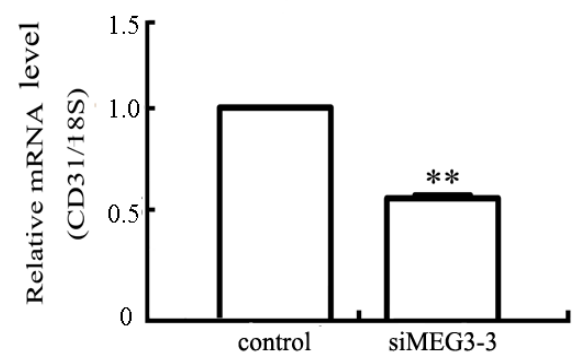

Figure 4. Knockdown of MEG3 reduced the mRNA level of CD31 induced by VEGF and bFGF. (A)The mRNA levels of MEG3 in hMSCs transfected with control siRNA or siMEG3. (B)The mRNA levels of CD31 in hMSCs transfected with siMEG3 in the presence of VEGF and bFGF at $7 \mathrm{~d}$.

\section{Conclusion}

It has been reported that MEG3 is a considerable conservation sequence between mouse and human and exhibits a tumor suppressive function [7]. The increased expression of some VEGF pathway genes and increased cortical microvessel density are observed in MEG3-null embryos, suggesting MEG3 may contribute to the regulation of vascularization [8-9]. Here, we found that the level of MEG3 was significantly increased in HUVECs, compared to hMSCs. The differentiated hMSCs exhibited increased expression of MEG3. Furthermore, silence of MEG3 using siRNA targeted against MEG3 can decrease the expression of CD31 induced by VEGF and bFGF. Thus, our data illustrated that MEG3 might play an important role in endothelial differentiation of hMSCs.

\section{Acknowledgments}

This work was financially supported by the National Natural Science Foundation of China (31171303, 31301073 and 31470816).

\section{References}

[1] M.F. Pittenger, A.M. Mackay, S.C. Beck, R.K. Jaiswal, R. Douglas, J.D. Mosca, M.A. Moorman, 
D.W. Simonetti, S. Craig, and D.R. Marshak. Science. 284(1999), p. 143-7

[2] J. Oswald, S. Boxberger, B. Jorgensen, S. Feldmann, G. Ehninger, M. Bornhauser, and C. Werner. Stem Cells. 22(2004), p. 377-84

[3] N. Wang, R. Zhang, S.J. Wang, C.L. Zhang, L.B. Mao, C.Y. Zhuang, Y.Y. Tang, X.G. Luo, H. Zhou, and T.C. Zhang. Int J Biochem Cell Biol. 45(2013), p. 1447-56

[4] F.E. Gordon, C.L. Nutt, P. Cheunsuchon, Y. Nakayama, K.A. Provencher, K.A. Rice, Y. Zhou, X. Zhang, and A. Klibanski. Endocrinology. 151(2010), p. 2443-52

[5] T.L. Young, T. Matsuda, and C.L. Cepko. Curr Biol. 15(2005), p. 501-12

[6] Y. Zhou, X. Zhang, and A. Klibanski. J Mol Endocrinol. 48(2012), p. R45-53

[7] K.M. Michalik, X. You, Y. Manavski, A. Doddaballapur, M. Zornig, T. Braun, D. John, Y. Ponomareva, W. Chen, S. Uchida, R.A. Boon, and S. Dimmeler. Circ Res. 114(2014), p. 1389-97

[8] M.H. Wei, N.C. Popescu, M.L. Lerman, M.J. Merrill, and D.B. Zimonjic. Hum Genet. 97(1996), p. $794-7$

[9] Y. Xu, L. Liu, Zhang, S. Fu, Y. Wang, H. Fu, K. Wu, H. Xiao, S. Liu, X. Yu, W. Zheng, B. Feng, and H. Huang. PLoS One. 7(2012), p.e34321 